Pacific Journal of Mathematics

ISOMETRIES BETWEEN INJECTIVE TENSOR PRODUCTS OF 


\title{
ISOMETRIES BETWEEN INJECTIVE TENSOR PRODUCTS OF BANACH SPACES
}

\author{
KRZYSZTOF JAROSZ
}

Let $K, H$ be real Banach spaces with strictly convex duals, and let $X, Y$ be any real Banach spaces. In this paper we find a general form of isometries between the Banach spaces $X \ddot{\otimes} K$ and $Y \ddot{\otimes} H$. As a consequence we obtain that $X \check{\otimes} K$ and $Y \ddot{\otimes} K$ are isometric if and only if $X$ and $Y$ are isometric. We also derive a theorem characterizing Banach spaces with a trivial centralizer.

0. Introduction. Let $X, Y, K, H$ be real Banach spaces. The purpose of this note is to study the isometries between the injective tensor products $X \check{\otimes} K$ and $Y \check{\otimes} H$. We find a general form of such isometries provided $K$ and $H$ have strictly convex duals, and using this characterization we investigate the following problems.

Problem 1. Under what conditions on $K, H$ or on $X, Y, K, H$ are the spaces $X \check{\otimes} K$ and $Y \check{\otimes} H$ isometric if and only if either the spaces $X, Y$ and $K, H$ or $X, H$ and $K, Y$ are isometric?

Problem 2. Under what conditions on $X, K$ is every isometry from $X \check{\otimes} K$ onto itself canonical?

We call an isometry $T$ from $X \check{\otimes} K$ onto $Y \check{\otimes} H$ canonical if one of the following possibilities holds:

(A) $T$ is of the form

$$
T(x \otimes k)=T_{1}(x) \otimes T_{2}(k) \text { for all } x \in X \text { and } k \in K,
$$

where $T_{1}: X \rightarrow Y, T_{2}: K \rightarrow H$ are onto isometries.

(B) There is a Banach space $Z$ such that $X$ is isometric to $Z \dot{\otimes} H$ and $Y$ is isometric to $Z \check{\otimes} K$, and under this identification $T$ is of the form

$$
T(z \otimes h \otimes k)=z \otimes k \otimes h \quad \text { for all } z \in Z, h \in H, k \in K .
$$

Notice that, in general, the implications in the problems do not hold. For example, take four compact Hausdorff spaces $S_{1}, \ldots, S_{4}$ which are pairwise non-homeomorphic but $S_{1} \times S_{2}$ and $S_{3} \times S_{4}$ are homeomorphic, and put $X_{i}=C\left(S_{i}\right)$ for $i=1, \ldots, 4$, where $C(S)$ is the space of all 
continuous functions on $S$ with sup-norm. We have

$$
X_{1} \check{\otimes} X_{2} \simeq C\left(S_{1} \times S_{2}\right) \simeq C\left(S_{2} \times S_{4}\right) \simeq X_{3} \check{\otimes} X_{4},
$$

but any two of the spaces $X_{i}, i=1, \ldots, 4$, are not isometric.

The special case of the above problems- $X=C(S), Y=C\left(S^{\prime}\right)$ - has been studied by many authors, the most important of which, for our purposes, is the monograph by Behrends ([1]), who proved, among other things, that if the centralizers $Z(K)$ and $Z(H)$ of Banach spaces $K$ and $H$, respectively, are both trivial and if the spaces $C(S) \check{\otimes} K$ and $C\left(S^{\prime}\right) \check{\otimes} H$ are isometric, then $K$ and $H$, and also $C(S)$ and $C\left(S^{\prime}\right)$, are isometric.

All fundamental results on centralizers and function module representations that we use are found in [1]. We use standard Banach space terminology. The set of extreme points of a convex set $C$ is denoted by ext $C$. For a Banach space $Z, B(Z)$ denotes the closed unit ball of $Z$. For Banach spaces $U, V$, we denote by $L(U, V)(K(U, V))$ the Banach space of all continuous (compact) linear operators from $U$ into $V$, and by $U \simeq V$ we mean that $U$ and $V$ are isometric. Throughout the paper we frequently view a Banach space $V$ as a subspace of $C\left(\operatorname{ext} B\left(V^{*}\right)\right)$ or $C\left(B\left(V^{*}\right)\right)$, where ext $B\left(V^{*}\right) \subset B\left(V^{*}\right)$ are equipped with the weak-*-topology. The space $X \check{\otimes} K$ is regarded as a subspace of $C\left(\operatorname{ext} B\left(X^{*}\right) \times \operatorname{ext} B\left(K^{*}\right)\right)$.

\section{The results.}

THEOREM 1. Let $X, Y, H, K$ be real Banach spaces and assume $H^{*}$ and $K^{*}$ are strictly convex. If $T$ is an isometry from $X \dot{\otimes} K$ onto $Y \check{\otimes} H$, then there are Banach spaces $Z$ and $X_{2}$ such that

$$
X \simeq(Z \check{\otimes} H) \oplus_{\infty} X_{2} \text { and } Y \simeq(Z \check{\otimes} K) \oplus_{\infty} X_{2},
$$

and, up to the above isometries, the operator $T$ is of the form

$$
T\left(z \otimes h \otimes k, x_{2} \otimes k_{2}\right)=\left(z \otimes k \otimes h, T_{2}\left(x_{2} \otimes k_{2}\right)\right),
$$

where

$$
T_{2}\left(x_{2} \otimes k_{2}\right)\left(x^{*} \otimes h^{*}\right)=x^{*}\left(x_{2}\right) \cdot h^{*}\left(\Phi\left(x^{*}\right)\left(k_{2}\right)\right),
$$

and $\Phi$ : ext $B\left(X_{2}^{*}\right) \rightarrow L(K, H)$ is an operator from ext $B\left(X_{2}^{*}\right)$ into the set of isometries from $\mathrm{K}$ onto $\mathrm{H}$.

Before proving Theorem 1 we formulate the following two theorems as corollaries. 
TheOREM 2. Let $X, Y, K$ be real Banach spaces and assume $K^{*}$ is strictly convex. Then

(a) $X \otimes K \simeq Y \otimes K$ if and only if $X \simeq Y$. If $K$ has the approximation property, then

(b) $K(X, K) \simeq K(Y, K)$ if and only if $X^{*} \simeq Y^{*}$.

Proof. Point (a) is an immediate consequence of Theorem 1, and to get (b) it is sufficient to notice that if $K$ has the approximation property, then $K(X, K) \simeq X^{*} \check{\otimes} K$.

The next theorem characterizes Banach spaces with a trivial centralizer.

THEOREM 3. For any real Banach space $X$ the following are equivalent:

(i) $\operatorname{dim} Z(X)=1$;

(ii) for any real Banach space $K$ with $K^{*}$ strictly convex, every isometry from $X \check{\otimes} K$ onto itself is canonical;

(iii) for any real Hilbert space $H$ every isometry from $X \check{\otimes} H$ onto itself is canonical;

(iv) for the two-dimensional real Hilbert space $\mathrm{H}_{2}$ every isometry from $\mathrm{X} \check{\otimes} \mathrm{H}_{2}$ onto itself is canonical.

Proof. To prove (i) $\Rightarrow$ (ii), assume $\operatorname{dim} Z(X)=1$, let $K$ be a real Banach space with a strictly convex dual, and let $T$ be an isometry from $X \check{\otimes} K$ onto itself. Notice that if $X$ were a direct sum, with the sup-norm, of two Banach spaces, then the orthogonal projections onto both components would be in the centralizer of $X$. Thus by Theorem 1 and our assumption we have two possibilities.

(1) There is a Banach space $Z$ such that $X \simeq Z \check{\otimes} K$, and up to this isometry $T$ is of the form (2), so it is canonical.

(2) There is a linear isometry $T_{1}$ from $X$ onto itself and an operator $T_{2}$ : ext $B\left(X^{*}\right) \rightarrow \operatorname{Isom}(K, K)$ such that $T$ is of the form

$$
T(x \otimes k)\left(x^{*} \otimes k^{*}\right)=T_{1}(x)\left(x^{*}\right)\left(T_{2}\left(x^{*}\right)(k)\right)\left(k^{*}\right)
$$

for any $k^{*}$ in $K^{*}$ and any $k$ in $K$. Then the operator $S_{k^{*}, k}: X \rightarrow X$, defined by

$$
\begin{aligned}
S_{k^{*}, k}(x)\left(x^{*}\right) & =T(x \otimes k)\left(\left(T_{1}^{-1}\right)^{*}\left(x^{*}\right) \otimes k^{*}\right) \\
& =x\left(x^{*}\right)\left(T_{2}\left(\left(T_{1}^{-1}\right)^{*}\left(x^{*}\right)\right)(k)\right)\left(k^{*}\right),
\end{aligned}
$$

is a multiplier on $X$. So by assumption $S_{k^{*}, k}$ is just the multiplication by a constant, for any $k^{*} \in K^{*}, k \in K$. Hence $T_{2}$ is a one-dimensional operator, and this means that $T$ is canonical of the form (1). 
The implications (ii) $\Rightarrow$ (iii) $\Rightarrow$ (iv) are trivial.

To prove (iv) $\Rightarrow$ (i), assume $Z(X)$ is not one-dimensional. Then there is a continuous, non-constant function $\varphi$ : ext $B\left(X^{*}\right) \rightarrow[0,2 \pi)$ such that the operator $M_{\varphi}: C\left(\operatorname{ext} B\left(X^{*}\right)\right) \rightarrow C\left(\operatorname{ext} B\left(X^{*}\right)\right): M_{\varphi}(f)=\varphi \cdot f$ leaves $X$ invariant. It is easy to check that the operator

$$
T: X \otimes H_{2} \rightarrow X \otimes H_{2}: T(x \otimes h)\left(x^{*} \otimes h^{*}\right)=x^{*}(x) \Phi\left(x^{*}\right)(h),
$$

where $\Phi\left(x^{*}\right): H_{2} \rightarrow H_{2}$ is the operator of rotation through angle $\varphi\left(x^{*}\right)$, is a well-defined, non-canonical onto isometry.

2. Proof of Theorem 1. The theorem is trivial when one of the spaces $K$ or $H$ is one dimensional, so we assume $\operatorname{dim} K \geq 2, \operatorname{dim} H \geq 2$. We start the proof with two propositions. The first is a special case of the theorem of Ruess and Stegall (it can also be found in Tseitlin's paper ([3])), and the second is a very easy, strictly algebraic fact.

THEOREM ([2], [3]). Let $X$ and $K$ be real Banach spaces. Then $\operatorname{ext} B\left((X \check{\otimes} K)^{*}\right)=\operatorname{ext} B\left(X^{*}\right) \otimes \operatorname{ext} B\left(K^{*}\right)$.

Proposition 1. Let $U$ and $V$ be linear spaces and assume $u_{1} \otimes v_{1}+u_{2}$ $\otimes v_{2}=u_{3} \otimes v_{3}$, where $u_{i} \in U, \quad v_{i} \in V, i=1,2,3$. Then the vectors $u_{1}, u_{2}, u_{3}$ or $v_{1}, v_{2}, v_{3}$ are proportional.

Proof. Let $v^{*}$ be any linear functional on $V$. We have

$$
u_{1} v^{*}\left(v_{1}\right)+u_{2} v^{*}\left(v_{2}\right)=u_{3} v^{*}\left(v_{3}\right) \text {. }
$$

Hence, if $v^{*}\left(v_{3}\right) \neq 0$, then $u_{3}$ is a linear combination of $u_{1}$ and $u_{2}$; if $u_{1}, u_{2}, u_{3}$ were not proportional, then the coefficients of this linear combination would be uniquely determined, and this would mean

$$
v^{*}\left(v_{1}\right)=\text { const } v^{*}\left(v_{3}\right), \quad v^{*}\left(v_{2}\right)=\text { const } v^{*}\left(v_{3}\right) \quad \text { for any } v^{*} \in V^{*} \text {. }
$$

Hence, $v_{1} \| v_{2}$ and $v_{2} \| v_{3}$.

Now let $X, Y, H, K, T$ be as in Theorem 1. Fix $y_{0}^{*} \in \operatorname{ext} B\left(Y^{*}\right)$, and let $h_{1}^{*}, h_{2}^{*}, h_{3}^{*}$ be any linearly independent elements of ext $B\left(H^{*}\right)$. By the Ruess-Stegall theorem

$$
T^{*}\left(\operatorname{ext} B\left(Y^{*}\right) \otimes \operatorname{ext} B\left(H^{*}\right)\right)=\operatorname{ext} B\left(X^{*}\right) \otimes \operatorname{ext} B\left(K^{*}\right),
$$

so there are $x_{1}^{*}, x_{2}^{*}, x_{3}^{*} \in \operatorname{ext} B\left(X^{*}\right)$ and $k_{1}^{*}, k_{2}^{*}, k_{3}^{*} \in \operatorname{ext} B\left(K^{*}\right)$ such that

$$
T^{*}\left(y_{0}^{*} \otimes h_{i}^{*}\right)=x_{i}^{*} \otimes k_{i}^{*} \quad \text { for } i=1,2,3 .
$$


Since $H^{*}$ is strictly convex, it follows that $\left(h_{1}^{*}+h_{2}^{*}\right) /\left\|h_{1}^{*}+h_{2}^{*}\right\|$ is an extreme point of $B\left(H^{*}\right)$, so

$$
x_{1}^{*} \otimes k_{1}^{*}+x_{2}^{*} \otimes k_{2}^{*}=T^{*}\left(y_{0}^{*} \otimes\left(h_{1}^{*}+h_{2}^{*}\right)\right)=\left\|h_{1}^{*}+h_{2}^{*}\right\| x_{4}^{*} \otimes k_{4}^{*}
$$

for some $x_{4}^{*} \in \operatorname{ext} B\left(X^{*}\right)$ and $k_{4}^{*} \in \operatorname{ext} B\left(K^{*}\right)$. Hence by Proposition 1 we have $x_{1}^{*} \| x_{2}^{*}$ or $k_{1}^{*} \| k_{2}^{*}$. The same arguments show that $x_{1}^{*} \| x_{3}^{*}$ or $k_{1}^{*} \| k_{3}^{*}$, and $x_{2}^{*} \| x_{3}^{*}$ or $k_{2}^{*} \| k_{3}^{*}$, and this proves that $x_{1}^{*}\left\|x_{2}^{*}\right\| x_{3}^{*}$ or $k_{1}^{*}\left\|k_{2}^{*}\right\| k_{3}^{*}$. The strict convexity of $H^{*}$ together with (4) now implies that, for any $y_{0}^{*} \in \operatorname{ext} B\left(Y^{*}\right)$, one of the following occurs:

(1) There is an element $k_{1}^{*}$ in ext $B\left(K^{*}\right)$ and a linear, weak-*-continuous into isometry $\Phi: H^{*} \rightarrow X^{*}$ such that

$$
T^{*}\left(y_{0}^{*} \otimes h^{*}\right)=\Phi\left(h^{*}\right) \otimes k_{1}^{*} \quad \text { for any } h^{*} \text { in } H^{*} .
$$

(2) There is an element $x_{2}^{*}$ of ext $B\left(X^{*}\right)$ and a linear, weak-*-continuous isometry $\Psi: H^{*} \rightarrow K^{*}$ such that

$$
T^{*}\left(y_{0}^{*} \otimes h^{*}\right)=x_{2}^{*} \otimes \Psi\left(h^{*}\right) \text { for any } h^{*} \text { in } H^{*} .
$$

Let us denote by $S_{1}$ the subset of ext $B\left(Y^{*}\right)$ consisting of all points $y^{*}$ for which the first possibility holds, and by $S_{2}$ the subset of ext $B\left(Y^{*}\right)$ for which the second holds. We have four functions:

$$
\begin{array}{ll}
\Phi_{1}: S_{1} \times H^{*} \rightarrow X^{*}, & \Psi_{1}: S_{1} \rightarrow \operatorname{ext} B\left(K^{*}\right), \\
\Phi_{2}: S_{2} \rightarrow \operatorname{ext} B\left(X^{*}\right), & \Psi_{2}: S_{2} \times H^{*} \rightarrow K^{*}
\end{array}
$$

such that for any $y^{*} \in S_{1}$

$$
T^{*}\left(y^{*} \otimes h^{*}\right)=\Phi_{1}\left(y^{*}, h^{*}\right) \otimes \Psi_{1}\left(y^{*}\right) \text { for all } h^{*} \in H^{*},
$$

and for any $y^{*} \in S_{2}$,

$$
T^{*}\left(y^{*} \otimes h^{*}\right)=\Phi_{2}\left(y^{*}\right) \otimes \Psi_{2}\left(y^{*}, h^{*}\right) \text { for all } h^{*} \in H^{*} .
$$

Using the same arguments for $T^{-1}$, we get that ext $B\left(X^{*}\right)$ is the sum of two disjoint subsets $\tilde{S}_{1}$ and $\tilde{S}_{2}$, and there are four functions:

$$
\begin{array}{ll}
\tilde{\Phi}_{1}: \tilde{S}_{1} \times K^{*} \rightarrow Y^{*}, & \tilde{\Psi}_{1}: \tilde{S}_{1} \rightarrow \operatorname{ext} B\left(H^{*}\right), \\
\tilde{\Phi}_{2}: \tilde{S}_{2} \rightarrow \operatorname{ext} B\left(Y^{*}\right), & \tilde{\Psi}_{2}: \tilde{S}_{2} \times K^{*} \rightarrow H^{*}
\end{array}
$$

such that for any $x^{*} \in \tilde{S}_{1}$

$$
\left(T^{-1}\right)^{*}\left(x^{*} \otimes k^{*}\right)=\tilde{\Phi}_{1}\left(x^{*}, k^{*}\right) \otimes \tilde{\Psi}_{1}\left(x^{*}\right) \text { for all } k^{*} \in K^{*},
$$

and for any $x^{*} \in \tilde{S}_{2}$

$$
\left(T^{-1}\right)^{*}\left(x^{*} \otimes k^{*}\right)=\tilde{\Phi}_{2}\left(x^{*}\right) \otimes \tilde{\Psi}_{2}\left(x^{*}, k^{*}\right) \text { for all } k^{*} \in K^{*} .
$$


It is easy to see that for any $y^{*} \in S_{2}$ the operator $\Psi_{2}\left(y^{*}, \cdot\right)$ is a weak-*-continuous isometry from $H^{*}$ onto $K^{*}$. Let $0 \neq y_{0}^{*} \in \bar{S}_{1}$ (the bar always denotes closure in the weak-*-topology), and let $\left(y_{\alpha}^{*}\right)_{\alpha \in \Gamma} \subset S_{1}$ be a net convergent to $y_{0}^{*}$; we can assume that the net $\left(\Psi_{1}\left(y_{\alpha}^{*}\right)\right)_{\alpha \in \Gamma}$ tends to $k_{0}^{*} \in K^{*}$. By (5) we have $k_{0}^{*} \neq 0$, and we get that the net $\left(\Phi_{1}\left(y_{\alpha}^{*}, h^{*}\right)\right)_{\alpha \in \Gamma}$ is convergent for any $h^{*} \in H^{*}$. Moreover,

$$
T^{*}\left(y_{0}^{*} \otimes h^{*}\right)=\lim _{\alpha} \Phi_{1}\left(y_{\alpha}^{*}, h^{*}\right) \otimes \lim _{\alpha} \psi_{1}\left(y_{\alpha}^{*}\right) \quad \text { for all } h^{*} \in H^{*} .
$$

By the same arguments applied to formulas (6)-(8), we get that the functions $\Phi_{i}, \tilde{\Phi}_{i}, \Psi_{i}, \tilde{\Psi}_{i}, i=1,2$, can be extended to the weak-*-closures of their domains, and formulas (5)-(8) remain valid for the extended functions. They will be denoted by the same letters. These functions are not uniquely determined by the formulas (5)-(8), and we will show that we can assume $\Phi_{2}^{-1}=\tilde{\Phi}_{2}$. To show this, let us notice that by applying (6) to the extended functions we get

$$
\begin{aligned}
\left\|y^{*}\right\|\left\|h^{*}\right\| & =\left\|y^{*} \otimes h^{*}\right\|=\left\|T^{*}\left(y^{*} \otimes h^{*}\right)\right\| \\
& =\left\|\Phi_{2}\left(y^{*}\right)\right\|\left\|\Psi_{2}\left(y^{*}, h^{*}\right)\right\| \\
& \quad \text { for all } h^{*} \in H^{*}, \text { for any } y^{*} \in \bar{S}_{2} .
\end{aligned}
$$

Hence, for any $y^{*} \in \bar{S}_{2}-\{0\}$ there is a $\lambda \in \mathbf{R}-\{0\}$ such that $\lambda \Psi_{2}\left(y^{*}, \cdot\right)$ is an isometry. We can define an equivalence relation on $\bar{S}_{2}-\{0\}$ by

$$
y_{1}^{*} \sim y_{2}^{*} \text { if } \Psi_{2}\left(y_{1}^{*}, \cdot\right)=\lambda \Psi_{2}\left(y_{2}^{*}, \cdot\right) \text { for some } \lambda \in \mathbf{R} \text {. }
$$

Multiplying the function $y^{*} \mapsto \Phi_{2}\left(y^{*}\right)$ by a scalar function, and the function $y^{*} \mapsto \Psi_{2}\left(y^{*}, \cdot\right)$ by its reciprocal, we can assume that both functions are constant in each equivalence class and, for $y^{*} \in \bar{S}_{2}-\{0\}$,

$$
\left\|\Phi_{2}\left(y^{*}\right)\right\|=\left\|y^{*}\right\| \text { and } \Psi_{2}\left(y^{*}, \cdot\right) \text { is an isometry. }
$$

By the same arguments $\tilde{\Phi}_{2}$ and $\tilde{\Psi}_{2}$ may have the same properties.

From (6) and (8) we get

$$
y^{*} \otimes h^{*}=\tilde{\Phi}_{2}\left(\Phi_{2}\left(y^{*}\right)\right) \otimes \tilde{\Psi}_{2}\left(\Phi_{2}\left(y^{*}\right), \Psi_{2}\left(y^{*}, h^{*}\right)\right)
$$

for any $y^{*} \in \overline{S_{2}}$ and all $h^{*} \in H^{*}$. Hence $y^{*} \| \tilde{\Phi}_{2}\left(\Phi_{2}\left(y^{*}\right)\right)$, so we get $y^{*}=\varepsilon \tilde{\Phi}_{2}\left(\Phi_{2}\left(y^{*}\right)\right)$, with $|\varepsilon|=1$. Similarly, for any $\lambda \in \mathbf{R}$, if $\lambda y^{*} \in \bar{S}_{2}$ we get

$$
\lambda y^{*}=\varepsilon_{\lambda} \tilde{\Phi}_{2}\left(\Phi_{2}\left(\lambda y^{*}\right)\right) \quad \text { with }\left|\varepsilon_{\lambda}\right|=1 \text {. }
$$

Hence, since the functions $x^{*} \mapsto \tilde{\Psi}_{2}\left(x^{*}, \cdot\right)^{*}$ and $y^{*} \mapsto \Psi_{2}\left(y^{*}, \cdot\right)$ are constant in each equivalence class, we get

$$
\tilde{\Psi}_{2}\left(\Phi_{2}\left(y^{*}\right), \Psi_{2}\left(y^{*}, \cdot\right)\right)=\tilde{\Psi}_{2}\left(\Phi_{2}\left(\lambda y^{*}\right), \Psi_{2}\left(\lambda y^{*}, \cdot\right)\right),
$$

so (9) gives $\varepsilon_{\lambda}=\varepsilon$ for all $\lambda \in \mathbf{R}$. 
The above proves that by multiplying $\Phi_{2}$, in any point of its domain, by +1 or -1 , depending on whether $\varepsilon=1$ or $\varepsilon=-1$, we get $\tilde{\Phi}_{2} \circ \Phi_{2}=\operatorname{Id}_{\bar{S}_{2}}$ and, by symmetry, $\Phi_{2}^{-1}=\tilde{\Phi}_{2}$.

We now put

$$
\begin{aligned}
& X_{i}=\left\{\left.f\right|_{\tilde{S}_{i}}: f \in X \subset C\left(\tilde{S}_{1} \cup \tilde{S}_{2}\right)\right\}, \\
& Y_{i}=\left\{\left.g\right|_{S_{t}}: g \in Y \subset C\left(S_{1} \cup S_{2}\right)\right\} \text { for } i=1,2 .
\end{aligned}
$$

We show that $X_{i}, Y_{i}, i=1,2$, are Banach spaces, and $X=X_{1} \oplus_{\infty} X_{2}$, $Y=Y_{1} \oplus_{\infty} Y_{2}$. First we study the spaces $X_{1}$ and $Y_{1}$.

\section{LEMMA 1.}

(i) For any $y_{1}^{*}, y_{2}^{*} \in S_{1}$ we have

$$
\Phi_{1}\left(y_{1}^{*}, \cdot\right)\left(H^{*}\right)=\Phi_{1}\left(y_{2}^{*}, \cdot\right)\left(H^{*}\right)
$$

or

$$
\Phi_{1}\left(y_{1}^{*}, \cdot\right)\left(H^{*}\right) \cap \Phi_{1}\left(y_{2}^{*}, \cdot\right)\left(H^{*}\right)=\{0\} .
$$

(ii) If $\Phi_{1}\left(y_{1}^{*}, \cdot\right)\left(H^{*}\right)=\Phi_{1}\left(y_{2}^{*}, \cdot\right)\left(H^{*}\right)$, then

$$
\Phi_{1}\left(y_{1}^{*}, \cdot\right)=\lambda \Phi_{1}\left(y_{2}^{*}, \cdot\right), \text { where } \lambda=+1 \text { or } \lambda=-1 \text {. }
$$

Proof. We first prove the following implication:

$$
\text { If } \Phi_{1}\left(y_{1}^{*}, h_{1}^{*}\right)=\Phi_{1}\left(y_{2}^{*}, h_{2}^{*}\right) \text {, then } y_{1}^{*} \| y_{2}^{*} \text { or } h_{1}^{*} \| h_{2}^{*} \text {. }
$$

For this purpose notice that, since $T^{*}$ is onto and $\Phi_{2}\left(S_{2}\right)=\tilde{S}_{2}$, there are $y_{3}^{*} \in S_{1}, h_{3}^{*} \in H^{*}$ such that

$$
T^{*}\left(y_{3}^{*} \otimes h_{3}^{*}\right)=\Phi_{1}\left(y_{1}^{*}, h_{1}^{*}\right) \otimes\left(\Psi_{1}\left(y_{1}^{*}\right)+\Psi_{1}\left(y_{2}^{*}\right)\right) .
$$

We have

$$
\begin{aligned}
T^{*}\left(y_{1}^{*} \otimes\right. & \left.h_{1}^{*}+y_{2}^{*} \otimes h_{2}^{*}\right) \\
& =\Phi_{1}\left(y_{1}^{*}, h_{1}^{*}\right) \otimes \Psi_{1}\left(y_{1}^{*}\right)+\Phi_{1}\left(y_{2}^{*}, h_{2}^{*}\right) \otimes \Psi_{1}\left(y_{2}^{*}\right) \\
& =T^{*}\left(y_{3}^{*} \otimes h_{3}^{*}\right) .
\end{aligned}
$$

Hence by Proposition 1 we get $h_{1}^{*} \| h_{2}^{*}$ or $y_{2}^{*} \| y_{3}^{*}$.

Now assume that $y_{1}^{*}, y_{2}^{*} \in S_{1}$, and $h_{0}^{*}, h_{1}^{*}, h_{2}^{*} \in H^{*}-\{0\}$ are such that $\Phi_{1}\left(y_{1}^{*}, h_{1}^{*}\right)=\Phi_{1}\left(y_{2}^{*}, h_{2}^{*}\right)$, but $\Phi_{1}\left(y_{1}^{*}, h_{0}^{*}\right) \notin \Phi_{1}\left(y_{2}^{*}, H^{*}\right)$. Let $y_{4}^{*}, y_{5}^{*}$ $\in S_{1} ; h_{4}^{*}, h_{5}^{*} \in H^{*}$ are such that

$$
\left\{\begin{array}{l}
T^{*}\left(y_{4}^{*} \otimes h_{4}^{*}\right)=\Phi_{1}\left(y_{1}^{*}, h_{0}^{*}\right) \otimes \Psi_{1}\left(y_{2}^{*}\right), \\
T^{*}\left(y_{5}^{*} \otimes h_{5}^{*}\right)=\Phi_{1}\left(y_{1}^{*}, h_{1}^{*}+h_{0}^{*}\right) \otimes \Psi_{1}\left(y_{2}^{*}\right) .
\end{array}\right.
$$


We have

$$
\begin{aligned}
T^{*}\left(y_{2}^{*} \otimes h_{2}^{*}+y_{4}^{*} \otimes h_{4}^{*}\right) \\
=\Phi_{1}\left(y_{2}^{*}, h_{2}^{*}\right) \otimes \Psi_{1}\left(y_{2}^{*}\right)+\Phi_{1}\left(y_{1}^{*}, h_{0}^{*}\right) \otimes \Psi_{1}\left(y_{2}^{*}\right) \\
=\Phi_{1}\left(y_{1}^{*}, h_{1}^{*}+h_{0}^{*}\right) \otimes \Psi_{1}\left(y_{2}^{*}\right)=T\left(y_{5}^{*} \otimes h_{5}^{*}\right) .
\end{aligned}
$$

Hence by Proposition 1 we have $y_{2}^{*} \| y_{4}^{*}$ or $h_{2}^{*} \| h_{4}^{*}$. By (10) and (11) we also have $h_{1}^{*} \| h_{2}^{*}$, and $h_{4}^{*} \| h_{0}^{*}$ or $y_{4}^{*} \| y_{1}^{*}$. Hence we have the following possibilities:

$1^{\circ} . h_{0}^{*} \| h_{1}^{*}$;

$2^{\circ} \cdot y_{2}^{*} \| y_{4}^{*}$

$3^{\circ} . y_{4}^{*} \| y_{1}^{*}$.

If $h_{0}^{*} \| h_{1}^{*}$, then

$$
\Phi_{1}\left(y_{1}^{*}, h_{0}^{*}\right) \| \Phi_{1}\left(y_{1}^{*}, h_{1}^{*}\right)=\Phi_{1}\left(y_{2}^{*}, h_{2}^{*}\right) \in \Phi_{1}\left(y_{2}^{*}, H^{*}\right),
$$

which contradicts our assumption. If $y_{2}^{*} \| y_{4}^{*}$, then $\Phi_{1}\left(y_{2}^{*}, H^{*}\right)=$ $\Phi_{1}\left(y_{4}^{*}, H^{*}\right)$, and by the assumption, $\Phi_{1}\left(y_{1}^{*}, h_{0}^{*}\right) \notin \Phi_{1}\left(y_{4}^{*}, H^{*}\right)$, which contradicts (11). If $y_{4}^{*} \| y_{1}^{*}$, then, since (11) implies $\Psi_{1}\left(y_{2}^{*}\right) \| \Psi_{1}\left(y_{4}^{*}\right)$, we get $\Psi_{1}\left(y_{1}^{*}\right) \| \Psi_{1}\left(y_{2}^{*}\right)$, and hence the vectors

$$
\begin{aligned}
& T^{*}\left(y_{1}^{*} \otimes h_{1}^{*}\right)=\Phi_{1}\left(y_{1}^{*}, h_{1}^{*}\right) \otimes \Psi_{1}\left(y_{1}^{*}\right) \text { and } \\
& T^{*}\left(y_{2}^{*} \otimes h_{2}^{*}\right)=\Phi_{1}\left(y_{2}^{*}, h_{2}^{*}\right) \otimes \Psi_{1}\left(y_{2}^{*}\right)
\end{aligned}
$$

are proportional, so $T^{*}$ being injective gives $y_{1}^{*} \| y_{2}^{*}$, which is impossible.

So we have proved (i). To end the proof of (ii), let us notice that if the images of the isometric embeddings $\Phi_{1}\left(y_{1}^{*}, \cdot\right)$ and $\Phi_{1}\left(y_{2}^{*}, \cdot\right)$ coincide, then, by (10), for any $h^{*} \in H^{*}$ there is a number $\lambda_{h^{*}}$ of modulus one such that

$$
\Phi_{1}\left(y_{1}^{*}, h^{*}\right)=\lambda_{h^{*}} \Phi_{1}\left(y_{2}^{*}, h^{*}\right) .
$$

So if we compose $\Phi_{1}\left(y_{1}^{*}, \cdot\right)$ with the inverse map to $\Phi_{1}\left(y_{2}^{*}, \cdot\right)$ (restricted to its image), we get the isometry $I$ from $H^{*}$ onto itself with the property that every element of $H^{*}$ is its eigenvector; hence $I=$ const - $\mathrm{Id}_{H^{*}}$, and this means that the function $h^{*} \mapsto \lambda_{h^{*}}$ is constant, and we get (ii).

For any $y^{*} \in S_{1}$, the operator $\Phi_{1}\left(y^{*}, \cdot\right)$ is weak-*-continuous, so $\left(\Phi_{1}\left(y^{*}, \cdot\right)\right)^{*}$ maps $X$ onto $H$. Let us denote the restriction of $\left(\Phi_{1}\left(y^{*}, \cdot\right)\right)^{*}$ to $X$ by $\Phi_{1}^{*}\left(y^{*}, \cdot\right)$, and let $\Omega$ denote the subset $\left\{\lambda \Phi_{1}^{*}\left(y^{*}, \cdot\right): y^{*} \in S_{1}\right.$, $\lambda= \pm 1\}$ of the space $L(X, H)$ equipped with the topology given by the family of seminorms

$$
\left\{L(X, H) \ni R \mapsto\left|h^{*}(R x)\right|: x \in X, h^{*} \in H^{*}\right\} .
$$


We define maps $Q$ and $Q_{1}$ :

$Q: S_{1} \rightarrow \Omega \otimes \operatorname{ext} B\left(K^{*}\right), \quad Q\left(y^{*}\right)=\Phi_{1}^{*}\left(y^{*}, \cdot\right) \otimes \Psi_{1}\left(y^{*}\right)$, $Q_{1}=Q \otimes \operatorname{Id}_{\text {ext } B\left(H^{*}\right)}: S_{1} \otimes \operatorname{ext} B\left(H^{*}\right) \rightarrow \Omega \otimes \operatorname{ext} B\left(K^{*}\right) \otimes \operatorname{ext} B\left(H^{*}\right)$, $Q_{1}\left(x^{*} \otimes h^{*}\right)=Q\left(y^{*}\right) \otimes h^{*}$.

By (5) the operators $Q$ and $Q_{1}$ are continuous and one-to-one, and by Lemma 1 they are onto.

To prove they are onto, it is sufficient to show that, for any $y_{0}^{*} \in S_{1}$ and $k_{0}^{*} \in \operatorname{ext} B\left(K^{*}\right)$, there is a $y^{*} \in S_{1}$ such that $\Phi_{1}\left(y_{0}^{*}, \cdot\right)=\varepsilon \Phi_{1}\left(y^{*}, \cdot\right)$ and $k_{0}^{*}=\varepsilon \Psi_{1}\left(y^{*}\right)$, where $|\varepsilon|=1$. Let $h_{0}^{*} \in \operatorname{ext} B\left(H^{*}\right)$. Since $T^{*}$ is onto, there is a $y^{*} \in S_{1}$ and $h^{*} \in \operatorname{ext} B\left(H^{*}\right)$ such that

$$
T^{*}\left(y^{*} \otimes h^{*}\right)=\Phi_{1}\left(y_{0}^{*}, h_{0}^{*}\right) \otimes k_{0}^{*} .
$$

On the other hand, from (5), we have

$$
T^{*}\left(y^{*} \otimes h^{*}\right)=\Phi_{1}\left(y^{*}, h^{*}\right) \otimes \Psi_{1}\left(h^{*}\right) .
$$

Hence $\Phi_{1}\left(y_{0}^{*}, H^{*}\right) \cap \Phi_{1}\left(y^{*}, H^{*}\right) \neq\{0\}$, and by Lemma 1 there is an $\varepsilon \in \mathbf{R},|\varepsilon|=1$, such that $\Phi_{1}\left(y_{0}^{*}, \cdot\right)=\varepsilon \Phi_{1}\left(y^{*}, \cdot\right)$. So by (12), (13) we get $k_{0}^{*}=\varepsilon \Psi_{1}\left(h^{*}\right)$.

By definition we have

$$
Q_{1}^{-1}\left(\omega \otimes k^{*} \otimes h^{*}\right)=\left(T^{-1}\right)^{*}\left(\omega\left(h^{*}\right) \otimes k^{*}\right),
$$

so $Q^{-1}$ and $Q_{1}^{-1}$ are also continuous.

Analogously we define $\tilde{\Phi}_{1}^{*}\left(x^{*}, \cdot\right) \in L(Y, K)$ for $x^{*} \in \tilde{S}_{1}$, the set $\tilde{\Omega}=\left\{\lambda \Phi_{1}^{*}\left(x^{*}, \cdot\right): x^{*} \in \tilde{S}_{1}, \lambda= \pm 1\right\}$, and two homeomorphisms $P$ and $P_{1}$ :

$$
\begin{aligned}
& P: \tilde{S}_{1} \rightarrow \tilde{\Omega} \otimes \operatorname{ext} B\left(H^{*}\right), \quad P\left(x^{*}\right)=\tilde{\Phi}_{1}^{*}\left(x^{*}, \cdot\right) \otimes \tilde{\Psi}_{1}\left(x^{*}\right), \\
& P_{1}=P \otimes \operatorname{Id}_{\operatorname{ext} B\left(K^{*}\right)}
\end{aligned}
$$

The maps $Q, Q_{1}, P$, and $P_{1}$ are homeomorphisms, so they define the isometric embeddings:

$$
\begin{aligned}
& Q^{0}: Y_{1} \mapsto C\left(\Omega \otimes \operatorname{ext} B\left(K^{*}\right)\right), \\
& Q_{1}^{0}: Y_{1} \otimes H \mapsto C\left(\Omega \otimes \operatorname{ext} B\left(K^{*}\right) \otimes \operatorname{ext} B\left(H^{*}\right)\right), \\
& P^{0}: X_{1} \mapsto C\left(\tilde{\Omega} \otimes \operatorname{ext} B\left(H^{*}\right)\right), \\
& P_{1}^{0}: X_{1} \otimes K \mapsto C\left(\tilde{\Omega} \otimes \operatorname{ext} B\left(H^{*}\right) \otimes \operatorname{ext} B\left(K^{*}\right)\right) .
\end{aligned}
$$

By (5) and (7) the homeomorphism $P_{1} \circ T^{*} \circ Q_{1}^{-1}$ is of the form $\Omega \otimes \operatorname{ext} B\left(K^{*}\right) \otimes \operatorname{ext} B\left(H^{*}\right) \ni \omega \otimes k^{*} \otimes h^{*}$

$$
\mapsto \varphi(\omega) \otimes h^{*} \otimes k^{*} \in \tilde{\Omega} \otimes \operatorname{ext} B\left(H^{*}\right) \otimes \operatorname{ext} B\left(K^{*}\right),
$$


where $\varphi$ is a homeomorphism from $\Omega$ onto $\tilde{\Omega}$. Hence, for any $h^{*} \in$ ext $B\left(H^{*}\right)$ and $k^{*} \in \operatorname{ext} B\left(K^{*}\right)$ we have

$$
\begin{aligned}
\left.\operatorname{Im} Q^{0}\right|_{\Omega \otimes\left\{k^{*}\right\}} & =\left.\left.\operatorname{Im} Q_{1}^{0}\right|_{\Omega \otimes\left\{k^{*}\right\} \otimes\left\{h^{*}\right\}} \simeq \operatorname{Im} P_{1}^{0}\right|_{\tilde{\Omega} \otimes\left\{h^{*}\right\} \otimes\left\{k^{*}\right\}} \\
& =\left.\operatorname{Im} P^{0}\right|_{\tilde{\Omega} \otimes\left\{h^{*}\right\}},
\end{aligned}
$$

so the space $\left.\operatorname{Im} Q^{0}\right|_{\Omega \otimes\left\{k^{*}\right\}}$ does not depend on the choice of $k^{*} \in$ ext $B\left(K^{*}\right)$, and we denote it by $Z$.

For any $h^{*}$ in ext $B\left(H^{*}\right)$ we have

$$
\left.Y_{1} \simeq \operatorname{Im} Q^{0} \simeq \operatorname{Im} P_{1}^{0}\right|_{\tilde{\Omega} \otimes\left\{h^{*}\right\} \otimes \operatorname{ext} B\left(K^{*}\right)} \subset Z \check{\otimes} K,
$$

and by the same arguments, for any $k^{*}$ in ext $B\left(K^{*}\right)$,

$$
\left.X_{1} \simeq \operatorname{Im} P^{0} \simeq \operatorname{Im} Q_{1}^{0}\right|_{\Omega \otimes\left\{k^{*}\right\} \otimes \operatorname{ext} B\left(H^{*}\right)} \subset Z \check{\otimes} H .
$$

So $Y_{1}\left(X_{1}\right)$ is isometric to a subspace of $Z \check{\otimes} K(Z \check{\otimes} H)$ which contains any element of the form $z \otimes k(z \otimes h)$ for $z \in Z, k \in K, h \in H$, and therefore, to end the proofs of $Y_{1} \simeq Z \dot{\otimes} K$ and $X_{1} \simeq Z \dot{\otimes} H$, it is sufficient to show that $Y_{1}, X_{1}$, and, as a consequence, $Z$, are complete. For the sake of simplicity of notation, we will assume from now on, without loss of generality, that $S_{1}=\Omega \otimes \operatorname{ext} B\left(K^{*}\right), \tilde{S}_{1}=\tilde{\Omega} \otimes \operatorname{ext} B\left(H^{*}\right), \varphi=$ $\mathrm{id}_{\Omega}, Z \otimes H \subset X_{1} \subseteq Z \otimes \dot{\otimes} H, Z \otimes K \subset Y_{1} \subseteq Z \otimes \check{\otimes} K$, and, consequently,

$$
\begin{array}{r}
T^{*}\left(\omega \otimes k^{*} \otimes h^{*}\right)=\omega \otimes h^{*} \otimes k^{*} \quad \text { for any } \omega \otimes k^{*} \in S_{1} \\
\text { and } h^{*} \in \operatorname{ext} B\left(H^{*}\right) .
\end{array}
$$

For any $h^{*} \in H^{*}$ and $k \in K$ we define a continuous, linear operator $S_{h^{*}, k}: X \rightarrow Y:$

$$
y^{*}\left(S_{h^{*}, k}(x)\right)=y^{*} \otimes h^{*}(T(x \otimes k)) \quad \text { for any } y^{*} \in \operatorname{ext} B\left(Y^{*}\right) .
$$

Similarly, for any $k^{*} \in K^{*}$ and $h \in H$ we define a continuous, linear operator $\tilde{S}_{k^{*}, h}: Y \rightarrow X$ :

$$
x^{*}\left(\tilde{S}_{k^{*}, h}(y)\right)=x^{*} \otimes k^{*}\left(T^{-1}(y \otimes h)\right) \quad \text { for any } x^{*} \in \operatorname{ext} B\left(X^{*}\right) .
$$

By (14) and (6) we have

$$
\begin{aligned}
y^{*}\left(S_{h^{*}, k}(x)\right) & \\
& = \begin{cases}\omega \otimes h^{*}(x) k^{*}(k) & \text { for } y^{*}=\omega \otimes k^{*} \in S_{1}, \\
\Phi_{2}\left(y^{*}\right)(x) \Psi_{2}\left(y^{*}, h^{*}\right)(k) & \text { for } y^{*} \in \bar{S}_{2},\end{cases}
\end{aligned}
$$


and by (14) and (8) we have

$$
\begin{aligned}
& x^{*}\left(\tilde{S}_{k^{*}, h}(y)\right) \\
& \quad= \begin{cases}\omega \otimes k^{*}(y) h^{*}(h) & \text { for } x^{*}=\omega \otimes h^{*} \in \tilde{S}_{1}, \\
\tilde{\Phi}_{2}\left(x^{*}\right)(y) \tilde{\Psi}_{2}\left(x^{*}, k^{*}\right)(h) & \text { for } x^{*} \in \overline{\tilde{S}}_{2} .\end{cases}
\end{aligned}
$$

By the above equalities, for any $x_{0}^{*}=\omega_{0} \otimes h_{0}^{*} \in \tilde{S}_{1}$ we have

$$
x_{0}^{*}\left(\tilde{S}_{k^{*}, h} \circ S_{h^{*}, k}(x)\right)=\omega_{0} \otimes h^{*}(x) k^{*}(k) h_{0}^{*}(h),
$$

and for any $x_{0}^{*} \in \overline{\tilde{S}}_{2}$ the equality $\Phi_{2} \circ \tilde{\Phi}_{2}=\operatorname{Id} \tilde{\tilde{S}}_{2}$ gives

$$
x_{0}^{*}\left(\tilde{S}_{k^{*}, h} \circ S_{h^{*}, k}(x)\right)=x_{0}^{*}(x) \Psi_{2}\left(\tilde{\Phi}_{2}\left(x_{0}^{*}\right), h^{*}\right)(k) \tilde{\Psi}_{2}\left(x_{0}^{*}, k^{*}\right)(h) .
$$

To prove that $X_{1}$ is complete and $X=X_{1} \oplus_{\infty} X_{2}$, we have to show that for any $x=\left(x_{1}, x_{2}\right) \in X$ we have $\left(x_{1}, 0\right) \in X$, and since the map $X \ni\left(x_{1}, x_{2}\right) \mapsto\left(x_{1}, 0\right) \in X$ is linear and continuous and $Z \otimes H$ (algebraic tensor product of $Z$ and $H$ ) is a dense subset of $X_{1}$, it is sufficient to show that $\left(z_{0} \otimes h_{0}, 0\right) \in X$ for any $z_{0} \in Z, h_{0} \in H$. For this purpose fix $x_{0}=\left(z_{0} \otimes h_{0}, x_{2}\right) \in X$ with $\left\|z_{0}\right\|=\left\|h_{0}\right\|=1$. We show that for any $\varepsilon>0$ there is a continuous operator $A: X \rightarrow X$ (which depends on $x_{0}$ and $\varepsilon$ ) such that $A x_{0}=\left(z_{0} \otimes h_{0}, x_{2}^{\prime}\right)$ with $\left\|x_{2}^{\prime}\right\| \leq \varepsilon$, and, hence, by completeness of $X$ we get $\left(z_{0} \otimes h_{0}, 0\right) \in X$. To this end fix $x_{0}^{*} \in \overline{\tilde{S}}_{0}$ and let

$h_{1}^{*} \in \operatorname{ext} B\left(H^{*}\right)$ be such that $h_{1}^{*}\left(h_{0}\right)=1$,

$k_{1} \in K$ be such that $\left\|k_{1}\right\|=1$ and

$\Psi_{2}\left(\tilde{\Phi}_{2}\left(x_{0}^{*}\right), h_{1}^{*}\right)\left(k_{1}\right)=0$ (such $k_{1}$ exists provided that $\operatorname{dim} K \geq 2$ ),

$k_{1}^{*} \in \operatorname{ext} B\left(K^{*}\right)$ be such that $k_{1}^{*}\left(k_{1}\right)=1$.

By (17) for any $x^{*}=\omega \otimes h^{*} \in \tilde{S}_{1}$ we have

$\omega \otimes h^{*}\left(\tilde{S}_{k_{1}^{*}, h_{0}} \circ S_{h_{1}^{*}, k_{1}}\left(x_{0}\right)\right)=\omega \otimes h_{1}^{*}\left(z_{0} \otimes h_{0}, x_{2}\right) k_{1}^{*}\left(k_{1}\right) h^{*}\left(h_{0}\right)$

$$
\begin{aligned}
& =\omega\left(z_{0}\right) h_{1}^{*}\left(h_{0}\right) h^{*}\left(h_{0}\right)=\omega\left(z_{0}\right) h^{*}\left(h_{0}\right) \\
& =\omega \otimes h^{*}\left(z_{0} \otimes h_{0}\right)=\omega \otimes h^{*}\left(x_{0}\right) ;
\end{aligned}
$$

this means

$$
\left.\tilde{S}_{k_{1}^{*}, h_{0}} \circ S_{h_{1}^{*}, k_{1}}\left(x_{0}\right)\right|_{\tilde{S}_{1}}=x_{0},
$$

and by (18) we get, by the same arguments,

$$
\left.\tilde{S}_{k_{1}^{*}, h_{0}} \circ S_{h_{1}^{*}, k_{1}}\left(x_{0}\right)\right|_{\tilde{\tilde{S}}_{2}}=f_{x_{0}^{*}} \cdot x_{0},
$$

where

$$
f_{x_{0}^{*}}\left(x^{*}\right)=\Psi_{2}\left(\tilde{\Phi}_{2}\left(x^{*}\right), h_{1}^{*}\right)\left(k_{1}\right) \tilde{\Psi}_{2}\left(x^{*}, k_{1}^{*}\right)\left(h_{0}\right)
$$


is a continuous function on $\overline{\tilde{S}}_{2}$, of norm not greater than one and such that $f_{x_{0}^{*}}\left(x_{0}^{*}\right)=0$. Hence, by the compactness of $\tilde{S}_{2}$, for any $\varepsilon>0$, by iterating the action of the operators $\tilde{S}_{k_{j}^{*}, h_{0}} \circ S_{h_{j}^{*}, k_{j}}$ for suitable $k_{j}^{*}, h_{j}^{*}$, and $k_{j}$, we get $x^{\prime} \in X$ such that

$$
\left.x^{\prime}\right|_{\tilde{S}_{1}}=\left.x_{0}\right|_{S_{1}} \text { and }\left|x^{*}\left(x^{\prime}\right)\right| \leq \varepsilon \quad \text { for any } x^{*} \in \overline{\tilde{S}}_{2} .
$$

So we have proven that $X_{1}$ and $Y_{1}$ are complete; we have actually proven even more-namely, that $X_{2}, Y_{2}$ are also complete and $X \simeq$ $X_{1} \oplus_{\infty} X_{2}, Y \simeq Y_{1} \oplus_{\infty} Y_{2}$. Thus to end the proof it is sufficient if we restrict ourselves to investigating the isometry $T$ between $X_{2} \otimes K$ and $Y_{2} \otimes H$. Without loss of generality we can assume that $X_{2}$ and $Y_{2}$ are subspaces of some function modules $\Pi_{\alpha \in \tilde{\Gamma}} X_{\alpha}$ and $\Pi_{\alpha \in \Gamma} Y_{\alpha}$, respectively, and that the identity embeddings $\pi_{X}: X_{2} \mapsto \Pi_{\alpha \in \tilde{\Gamma}} X_{\alpha}$ and $\pi_{Y}: Y_{2} \mapsto$ $\Pi_{\alpha \in \Gamma} Y_{\alpha}$ give the maximal function module representation ([2]). Hence any $y^{*} \in S_{2}$ is of the form

$$
\begin{aligned}
\prod_{\alpha \in \Gamma} Y_{\alpha} \supset Y \ni y \stackrel{\delta_{\alpha} \otimes y_{\alpha}^{*}}{\mapsto} y_{\alpha}^{*}(y(\alpha)) \\
\quad \text { for some } y_{\alpha}^{*} \in \operatorname{ext} B\left(Y_{\alpha}^{*}\right) \text { and } \alpha \in \Gamma .
\end{aligned}
$$

Let $k \in K, h \in H, k^{*} \in K^{*}, h^{*} \in H^{*}$. By (18) the operator $\tilde{S}_{k^{*}, h} \circ S_{h^{*}, k}: X \rightarrow X$ is of the form

$$
\delta_{\alpha} \otimes x_{\alpha}^{*}\left(\tilde{S}_{k^{*}, h} \circ S_{h^{*}, k}\left(\left(x_{\alpha}\right)_{\alpha \in \Gamma}\right)\right)=f\left(\alpha \otimes x_{\alpha}^{*}\right) \cdot x_{\alpha}^{*}\left(x_{\alpha}\right),
$$

where

$$
f\left(\alpha \otimes x_{\alpha}^{*}\right)=\Psi_{2}\left(\tilde{\Phi}_{2}\left(\delta_{\alpha} \otimes x_{\alpha}^{*}\right), h^{*}\right)(k) \tilde{\Psi}_{2}\left(\delta_{\alpha} \otimes x_{\alpha}^{*}, k^{*}\right)(h),
$$

so it is just multiplication by a function $f: \tilde{S}_{2} \rightarrow \mathbf{R}$. Since we have the maximal function module representation, the function $f$ does not depend on $x_{\alpha}^{*}$ but only on $\alpha \in \Gamma$, and consequently the functions

$$
\tilde{S}_{2} \ni \delta_{\alpha} \otimes x_{\alpha}^{*} \mapsto \tilde{\Phi}_{2}\left(\delta_{2} \otimes x_{\alpha}^{*}\right)
$$

and

$$
\tilde{S}_{2} \ni \delta_{\alpha} \otimes x_{\alpha}^{*} \mapsto \tilde{\Psi}_{2}\left(\delta_{\alpha} \otimes x_{\alpha}^{*}, \cdot\right)
$$

also do not depend on $x_{\alpha}^{*}$ but only on $\alpha \in \Gamma$. Hence by (8) the operator $\left(T^{-1}\right)^{*}$ is of the form

$$
\begin{aligned}
\left(T^{-1}\right)^{*}\left(\delta_{\alpha} \otimes x_{\alpha}^{*} \otimes k^{*}\right) & =\tilde{\Phi}_{2}\left(\delta_{\alpha} \otimes x_{\alpha}^{*}\right) \otimes \tilde{\Psi}_{2}\left(\delta_{\alpha} \otimes x_{\alpha}^{*}, k^{*}\right) \\
& =\delta_{\varphi(\alpha)} \otimes \tilde{\Phi}_{\alpha}\left(x_{\alpha}^{*}\right) \otimes \tilde{\Psi}_{\alpha}\left(k^{*}\right),
\end{aligned}
$$

where $\varphi: \Gamma \rightarrow \tilde{\Gamma}$ and $\tilde{\Phi}_{\alpha}: X_{\alpha}^{*} \rightarrow Y_{\alpha}^{*}, \tilde{\Psi}_{\alpha}: K^{*} \rightarrow H^{*}$ are weak-*-continuous onto isometries. 
Composing the above formula with an analogous formula for $T^{*}$, we get

$$
\delta_{\alpha} \otimes x_{\alpha}^{*} \otimes k^{*}=\delta_{\psi \circ \varphi(\alpha)} \otimes\left(\Phi_{\varphi(\alpha)} \circ \tilde{\Phi}_{\alpha}\left(x_{\alpha}^{*}\right)\right) \otimes\left(\Psi_{\varphi(\alpha)} \circ \tilde{\Psi}_{\alpha}\left(k^{*}\right)\right) .
$$

Hence $\varphi$ is a bijection between $\Gamma$ and $\tilde{\Gamma}$, and we can assume $\Gamma=\tilde{\Gamma}$, $\varphi=\mathrm{id}_{\Gamma}$, and $T^{*}$ is of the form

$$
T^{*}\left(\delta_{\alpha} \otimes y_{\alpha}^{*} \otimes h^{*}\right)=\delta_{\alpha} \otimes \phi_{\alpha}\left(y_{\alpha}^{*}\right) \otimes \Psi_{\alpha}\left(h^{*}\right),
$$

where $\Phi_{\alpha}: Y_{\alpha}^{*} \rightarrow X_{\alpha}^{*}, \Psi_{\alpha}: H^{*} \rightarrow K^{*}$ are weak-*-continuous onto isometries.

Put

$$
A=\prod_{\alpha \in \Gamma} \Phi_{\alpha}^{*}: \prod_{\alpha \in \Gamma} X_{\alpha} \rightarrow \prod_{\alpha \in \Gamma} Y_{\alpha} .
$$

The operator $A$ is an onto isometry, and to conclude the proof we show $A(X)=Y$. By (19) and (16) for any $h^{*} \in \operatorname{ext} B\left(H^{*}\right), k \in K$ the operator $\tilde{S}_{k^{*}, h} \circ A: A^{-1}(Y) \rightarrow X$ is of the form

$$
x_{\alpha}^{*}\left(\tilde{S}_{k^{*}, h} \circ A(w)(\alpha)\right)=x_{\alpha}^{*}(w(\alpha)) \cdot \tilde{\Psi}_{\alpha}\left(k^{*}\right)(h) .
$$

Hence the function $\Gamma \ni \alpha \mapsto \tilde{\Psi}_{\alpha}\left(k^{*}\right)(h)$ is continuous, and, since $A^{-1}(Y)$ $\subset \prod_{\alpha \in \Gamma} X_{\alpha}$ is a function module, we get

$$
\tilde{S}_{k^{*}, h} \circ A(w) \in X \cap A^{-1}(Y)
$$

for any $w \in A^{-1}(Y), k^{*} \in \operatorname{ext} B\left(K^{*}\right), h \in H$.

So to prove $A^{-1}(Y) \subset X$ and, by symmetry, $A^{-1}(Y)=X$, it is sufficient to show that the set

$$
\operatorname{Lin}\left\{\tilde{S}_{k^{*}, h} \circ A(w): w \in A^{-1}(Y), k^{*} \in \operatorname{ext} B\left(K^{*}\right), h \in H\right\}
$$

is dense in $A^{-1}(Y)$, but this is an immediate consequence of the definition of $\tilde{S}_{k^{*}, h}$ :

$$
x^{*}\left(\tilde{S}_{k^{*}, h}(y)\right)=x^{*} \otimes k^{*}\left(T^{-1}(y \otimes h)\right)
$$

Hence

$$
x *\left(\sum_{j} \tilde{S}_{k^{*}, h_{j}}\left(y_{j}\right)\right)=x^{*} \otimes k^{*}\left(T^{-1}\left(\sum_{j} y_{j} \otimes h_{j}\right)\right),
$$

and the set $\left\{\sum_{j} y_{j} \otimes h_{j}: y_{j} \in Y, h_{j} \in H\right\}$ is dense in $Y \check{\otimes} H$, and $T^{-1}$ is onto.

REMARK. As proved by E. Behrends in the special case of Theorem 1 when $X=C(S)$ and $Y=C\left(S^{\prime}\right)$, the assumption about $K, H$ can be weakened to effect $\operatorname{dim} Z(H)=1=\operatorname{dim} Z(K)$. It is worthwhile to mention that, in general, this strengthened form of Theorem 1 is not valid: to 
provide an example, let $A$ be the disc algebra, i.e., the complex Banach algebra of all continuous functions defined on the unit disc on the complex plane which are analytic in the interior of the disc, and let $A_{R}$ denote the Banach space $A$ over the field of real numbers. Put $A_{R}^{j}$ for the injective tensor product of $j$ copies of $A_{R}$. We have $\operatorname{dim} Z\left(A_{R}^{j}\right)=1$ for $j=1,2, \ldots$ and

$$
A_{R}^{2} \otimes A_{R}^{3} \simeq A_{R}^{1} \otimes A_{R}^{4}
$$

while

$$
A_{R}^{j} \simeq A_{R}^{i} \text { only if } j=i \text {. }
$$

\section{REFERENCES}

[1] E. Behrends, M-Structure and the Banach-Stone Theorem, Lecture Notes in Math. 736, Springer, Berlin, Heidelberg, New York, 1979.

[2] W. M. Ruess and Ch. P. Stegall, Extreme points in duals of operator spaces, to appear in Math. Ann.

[3] I. I. Tseitlin, The extreme points of the unit balls of certain spaces of operators, Mat. Zametki, 20 (1976), 521-527.

Received May 10, 1983 and in revised form April 14, 1984.

WARSAW UNIVERSITY, PKIN,

00-901 WARSAW, POLAND 


\title{
PACIFIC JOURNAL OF MATHEMATICS EDITORS
}

V. S. VARADARAJAN (Managing Editor)
University of California
Los Angeles, CA 90024
HEBERT ClEMENS
University of Utah
Salt Lake City, UT 84112
CHARLES R. DEPRIMA
California Institute of Technology
Pasadena, CA 91125

R. FINN

Stanford University

Stanford, CA 94305

HERMANN FLASCHKA

University of Arizona

Tucson, AZ 85721

Ramesh A. Gangolli

University of Washington

Seattle, WA 98195

ROBION KIRBY

University of California

Berkeley, CA 94720
C. C. MOORE

University of California

Berkeley, CA 94720

H. SAMELSON

Stanford University

Stanford, CA 94305

HaRold Stark

University of California, San Diego

La Jolla, CA 92093

\section{ASSOCIATE EDITORS}

\author{
R. ARENS \\ E. F. BECKENBACH \\ B. H. NeumanN \\ F. Wolf \\ K. YoSHIDA \\ (1906-1982)
}

\section{SUPPORTING INSTITUTIONS}

UNIVERSITY OF ARIZONA
UNIVERSITY OF BRITISH COLUMBIA
CALIFORNIA INSTITUTE OF TECHNOLOGY
UNIVERSITY OF CALIFORNIA
MONTANA STATE UNIVERSITY
UNIVERSITY OF NEVADA, RENO
NEW MEXICO STATE UNIVERSITY
OREGON STATE UNIVERSITY

UNIVERSITY OF OREGON

UNIVERSITY OF SOUTHERN CALIFORNIA

STANFORD UNIVERSITY

UNIVERSITY OF HAWAII

UNIVERSITY OF TOKYO

UNIVERSITY OF UTAH

WASHINGTON STATE UNIVERSITY UNIVERSITY OF WASHINGTON

The Supporting Institutions listed above contribute to the cost of publication of this Journal, but they are not owners or publishers and have no responsibility for its content or policies.

Mathematical papers intended for publication in the Pacific Journal of Mathematics should be in typed form or offset-reproduced (not dittoed), double spaced with large margins. Please do not use built up fractions in the text of the manuscript. However, you may use them in the displayed equations. Underline Greek letters in red, German in green, and script in blue. The first paragraph must be capable of being used separately as a synopsis of the entire paper. In particular it should contain no bibliographic references. Please propose a heading for the odd numbered pages of less than 35 characters. Manuscripts, in triplicate, may be sent to any one of the editors. Please classify according to the scheme of Math. Reviews, Index to Vol. 39. Supply name and address of author to whom proofs should be sent. All other communications should be addressed to the managing editor, or Elaine Barth, University of California, Los Angeles, California 90024.

There are page-charges associated with articles appearing in the Pacific Journal of Mathematics. These charges are expected to be paid by the author's University, Government Agency or Company. If the author or authors do not have access to such Institutional support these charges are waived. Single authors will receive 50 free reprints; joint authors will receive a total of 100 free reprints. Additional copies may be obtained at cost in multiples of 50 .

The Pacific Journal of Mathematics is issued monthly as of January 1966. Regular subscription rate: $\$ 190.00$ a year (5 Vols., 10 issues). Special rate: $\$ 95.00$ a year to individual members of supporting institutions.

Subscriptions, orders for numbers issued in the last three calendar years, and changes of address should be sent to Pacific Journal of Mathematics, P.O. Box 969, Carmel Valley, CA 93924, U.S.A. Old back numbers obtainable from Kraus Periodicals Co., Route 100, Millwood, NY 10546.

The Pacific Journal of Mathematics at P.O. Box 969, Carmel Valley, CA 93924 (ISSN 0030-8730) publishes 5 volumes per year. Application to mail at Second-class postage rates is pending at Carmel Valley, California, and additional mailing offices. Postmaster: Send address changes to Pacific Journal of Mathematics, P.O. Box 969, Carmel Valley, CA 93924.

\section{PUBLISHED BY PACIFIC JOURNAL OF MATHEMATICS, A NON-PROFIT CORPORATION}

Copyright $\subset 1986$ by Pacific Journal of Mathematics 


\section{Pacific Journal of Mathematics}

\section{Vol. 121, No. 2 December, 1986}

Jorge Almeida, Minimal nonpermutative pseudovarieties of semigroups.

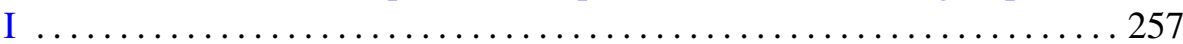

Jorge Almeida, Minimal nonpermutative pseudovarieties of semigroups.

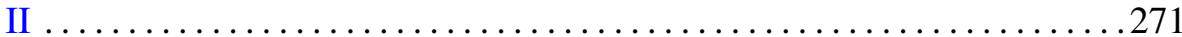

Carlos Andradas Heranz and José Manuel Gamboa Mutuberría, On

projections of real algebraic varieties $\ldots \ldots \ldots \ldots \ldots \ldots \ldots \ldots \ldots 281$

Zeev Ditzian, Inverse theorems for multidimensional Bernstein operators . . 293

M. Furi and Maria Patrizia Pera, A continuation principle for forced oscillations on differentiable manifolds $\ldots \ldots \ldots \ldots \ldots \ldots \ldots \ldots \ldots . \ldots . \ldots . \ldots 321$

James J. Hebda, The collars of a Riemannian manifold and stable

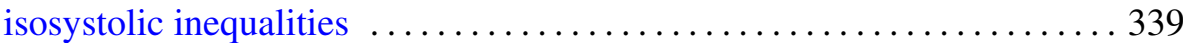

Henryk Hecht and Dragan Miličić, Character identities and asymptotic behavior of matrix coefficients of discrete series ................ 357

Piotr Jakóbczak, The boundary regularity of the solution of the $\bar{\partial}$-equation in the product of strictly pseudoconvex domains $\ldots \ldots \ldots \ldots \ldots \ldots . \ldots 371$

Krzysztof Jarosz, Isometries between injective tensor products of Banach

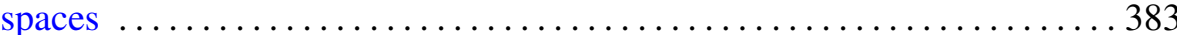

Hans Keller, On valued, complete fields and their automorphisms ........ 397

David Masser and Peter Man-Kit Shiu, On sparsely totient numbers . . . . 407

Tze-Beng Ng, Vector bundles over $(8 k+3)$-dimensional manifolds

Thomas Joseph Ransford, The spectrum of an interpolated operator and analytic multivalued functions

Akihito Uchiyama, On the radial maximal function of distributions 467 Jang-Mei Gloria Wu, On singularity of harmonic measure in space 